https://helda.helsinki.fi

\title{
Incidence of lichen sclerosus and subsequent causes of death: a nationwide Finnish register study
}

\section{Halonen, Pia}

2020-05

Halonen , P , Jakobsson, M , Heikinheimo , O , Gissler , M \& Pukkala , E 2020 , ' Incidence of lichen sclerosus and subsequent causes of death: a nationwide Finnish register study ' BJOG : An International Journal of Obstetrics \& Gynaecology , vol. 127 , no. 7 , pp. 814-819 . https://doi.org/10.1111/1471-0528.16175

http://hdl.handle.net/10138/326596

https://doi.org/10.1111/1471-0528.16175

unspecified

acceptedVersion

Downloaded from Helda, University of Helsinki institutional repository.

This is an electronic reprint of the original article.

This reprint may differ from the original in pagination and typographic detail.

Please cite the original version. 
DR. PIA HALONEN (Orcid ID : 0000-0002-5434-5069)

Article type : Main research article

\section{Incidence of lichen sclerosus and subsequent causes of death: a nationwide Finnish register study}

Halonen Pia ${ }^{1,2}$, Jakobsson Maija ${ }^{1,2}$, Heikinheimo Oskari ${ }^{2,3}$, Gissler Mika ${ }^{4,5}$, Pukkala Eero ${ }^{6,7}$

${ }^{1}$ Department of Obstetrics and Gynecology, HUCH Hyvinkää Hospital, Hyvinkää, Finland

${ }^{2}$ Department of Obstetrics and Gynecology, University of Helsinki, Helsinki, Finland.

${ }^{3}$ Department of Obstetrics and Gynecology, Helsinki University Hospital, Helsinki, Finland.

${ }^{4}$ Information Services Department, THL Finnish Institute for Health and Welfare, Helsinki, Finland.

${ }^{5}$ Karolinska Institutet, Department of Neurobiology, Care Sciences and Society, Division of Family Medicine, Stockholm, Sweden.

${ }^{6}$ Finnish Cancer Registry, Institute for Statistical and Epidemiological Cancer Research, Helsinki, Finland.

${ }^{7}$ Faculty of Social Sciences, Tampere University, Tampere, Finland.

Corresponding author: Pia Halonen, HUCH Hyvinkää Hospital, Department of Obstetrics and Gynecology, Sairaalankatu 1, FI-05850 Hyvinkää, HUCH, Finland. +358505345561, pia.halonen@helsinki.fi

Shortened title: Incidence and mortality in lichen sclerosus

This article has been accepted for publication and undergone full peer review but has not been through the copyediting, typesetting, pagination and proofreading process, which may lead to differences between this version and the Version of Record. Please cite this article as doi: $\underline{10.1111 / 1471-0528.16175}$

This article is protected by copyright. All rights reserved 


\section{Abstract \\ Objective}

To assess the incidence of lichen sclerosus (LS) in women and all-cause and cause-specific mortality of women with LS.

\section{Design}

Population-based descriptive study.

\section{Setting}

Finland.

\section{Population}

All Finnish women, including 7,790 women diagnosed with LS during 1969-2012.

\section{Methods}

Information gathered from the national Hospital Discharge Registry on women with LS is combined with dates and causes of death from Statistics Finland and Finnish Cancer Registry. Population statistics are from Statistics Finland.

\section{Main Outcome Measures}

Crude and age-adjusted incidence rates of LS and standardized mortality ratios (SMR).

\section{Results}

The incidence rate of LS adjusted for age (European Standard Population) increased from 14 per 100,000 woman-years in 2003 to 22 per 100,000 woman-years in 2010-2012. The agespecific incidence rate was highest in postmenopausal women (24-53 per 100,000), and was also elevated in girls in ages of 5-9 (7 per 100,000). All-cause mortality of women with LS was lower than in the general female population (SMR 0.84, 95\% confidence interval [95\% CI] 0.78-0.90), mostly due to decreased mortality from circulatory diseases (SMR 0.80, 95\% CI 0.72-0.89), and dementia and Alzheimer's disease (SMR 0.75, 95\% CI 0.62-0.88). Cancer mortality equaled that of the population, but vulvar cancer mortality was increased (SMR 28.1, 95\% CI 19.3-39.4).

\section{Conclusions}

LS is a common disease of elderly women. The overall mortality is decreased whereas mortality due to vulvar cancer is increased.

\section{Funding}


The Finnish-Norwegian Medical Foundation, Research Funds of the Hospital system of Helsinki and Uusimaa

\section{Keywords}

Lichen sclerosus, incidence, mortality, cause of death, epidemiology, prognosis

\section{Tweetable abstract}

The likelihood of getting LS by 80 is $1.6 \%$. The mortality of LS women is reduced compared to the population. 


\section{Introduction}

Lichen sclerosus (LS) is a chronic dermatosis commonly affecting vulvar skin. The etiology of the disease remains uncertain, but the prevailing theory suggests an autoimmune process inducing local inflammation of skin in the genital area. ${ }^{1}$

LS is most often diagnosed in postmenopausal women, but also in premenarcheal girls. ${ }^{2,3}$ The exact prevalence and incidence of LS are unknown because they have been studied in restricted populations. The reported prevalence rates have varied between two and seven per cent in gynecological units. ${ }^{4,5}$ An age-adjusted incidence rate of 16 per 100,000 womanyears was estimated with data from two Dutch provinces between 2006 and 2011. ${ }^{6}$

The most common symptom of LS is itch. Typical clinical findings are white patches or plaques with edema, fissuring, and atrophy in the genital area. Long-term sequelae of untreated LS include anatomical distortions, sexual dysfunction, decreased quality of life, and increased risk of vulvar cancer. ${ }^{7-9}$

Topical ultrapotent corticosteroid ointment is the current treatment of choice. It controls symptoms, improves clinical signs, and may reduce the risk of malignant transformation. ${ }^{10,11}$ Unfortunately, local corticosteroid treatment does not cure the disease, which typically follows a life-long course of remission and relapse. The effect of this chronic disease on mortality of patients is not known.

The aim of this study is to assess the incidence of LS in women using nation-wide data from Finland. In addition, we evaluated the all-cause and cause-specific mortality of women with LS.

\section{Methods}

This is a descriptive study based of all registered LS diagnoses of women in Finland between 1969 and 2012. The source of the LS diagnoses is the nationwide Hospital Discharge Registry (HDR) maintained by the Finnish Institute for Health and Welfare. In the HDR, data on inpatient diagnoses from 1969 as well as data on outpatient diagnoses made in public 
hospitals from 1998 onwards are included. The diagnoses in the HDR are recorded with the International Classification of Diseases (ICD) system, and the following codes were used to identify the cohort: 701.01 Lichen sclerosus et atrophicus (ICD-8), 7010B Lichen sclerosus et atrophicus (ICD-9) and L90.0 Lichen sclerosus (ICD-10). For identification of the women with LS in other registries, we used the individual personal identity code assigned to all Finnish citizens.

For incidence calculations, only women diagnosed within the last ten years of the study period, i.e. 2003-2012, were included. The crude incidence rate of LS was calculated by dividing the number of new LS diagnoses by the size of the Finnish female population. Population data were obtained from Statistics Finland. The European Standard Population 2013 and the World Standard Population were used to obtain age-adjusted incidence rates. The incidence rates were calculated in five-year age groups.

For calculations of mortality after LS diagnosis, all LS patients with a hospital visit between 1969 and 2012 were included. The follow-up started at the first hospital visit with LS, or on January 1, 1971 (whichever was later), and ended at the earliest date of the following: emigration, death, or on December 31, 2014. The dates for emigration and death were retrieved from the Population Register Centre. There were no women, who had both been diagnosed with LS and died between 1969 and 1971.

Causes of death were retrieved from Statistics Finland, which has gathered these data since 1936. The treating physician defines the cause of death using the relevant version of the ICD. To facilitate comparisons of these data over time, Statistics Finland has produced a 54category time series classification since 1971. We used this classification for all causes of death except those arising from cancers.

Cancer mortality data is from the Finnish Cancer Registry (FCR), which has gathered information on all cancer diagnoses in Finland since 1953.12 The FCR receives information on deaths from Statistics Finland and reassesses whether a specific cancer was the underlying cause of death of a cancer patient. 
Mortality was assessed using the standardized mortality ratio (SMR), which was calculated as a ratio of observed deaths to expected deaths. The observed deaths and person-years of follow-up were stratified by five-year age groups, calendar periods, and follow-up periods (less than five years, and five years or more since the LS diagnosis). The expected numbers of deaths were calculated by multiplying person-years in each stratum by the corresponding mortality rate in the Finnish female population. For non-cancer deaths, the reference rates were taken from Statistics Finland and for cancer deaths from the FCR. In defining the confidence intervals (CI), we assumed a Poisson distribution of the number of observed deaths.

We obtained the general study permission from the Finnish Institute for Health and Welfare (THL/1440/5.05.00/2013) and permission for the cause-of-death data from Statistics Finland (TK-53-1712-17). There was no direct patient or public involvement in this study. The study was supported by the Finnish-Norwegian Medical Foundation and the Research Funds of the Hospital system of Helsinki and Uusimaa. The funding organizations had no role in study design, data collection and analysis, decision to publish, or preparation of the manuscript.

\section{Results}

In Finland, 7,790 women visited a public hospital with an LS diagnosis between 1969 and 2012 (Table 1).

Incidence calculations include 5,258 patients from 2003-2012. The crude incidence rates and the rates adjusted for age according to the European Standard 2013 Population were similar (Figure 1). The incidence rate showed a slightly increasing trend from 14 per 100,000 woman-years in 2003 to 22 per 100,000 woman-years in 2010 where it stabilized thereafter (Figure 1). The age-adjusted incidence rate for the whole period of 2003-2012 was 19 per 100,000 woman-years when adjusted to the European Standard Population (Figure 1), and 12 per 100,000 woman-years when adjusted to the World Standard Population (Figure S1). 
A great majority of LS diagnoses were made in the postmenopausal age groups. The highest incidence rate (53 per 100,000 woman-years) was found in women aged from 75 to 79 years (Figure 2). There was a small elevation in incidence rate in girls from five to nine: 7 per 100,000 person-years. The cumulative incidence of LS by age 80 years was $1.6 \%$.

For SMR assessment all 7,790 women with LS were included. The follow-up of the women added up to 68,666 woman-years resulting in a mean follow-up time of 8.8 years (Table 1). Most deaths in the cohort were due to circulatory diseases, malignancies, and dementia and Alzheimer's disease.

The SMRs in all cause-of-death categories were consistently lower within the first five years after a diagnosis of LS than after five years, indicating a healthy patient selection bias (Table S1). Therefore, only the results for the follow-up excluding the five first years are presented in Table 2.

The overall mortality of the cohort was decreased (SMR 0.84, 95\% CI 0.78-0.90) (Table 2). Reduced mortality was found from the diseases of the circulatory system (SMR 0.80, 95\% CI 0.72-0.89) and from some of its more specific subcategories, e.g., ischemic and other heart diseases (Table 2). The risk of death from dementia and Alzheimer's disease was also decreased (SMR 0.75, 95\% CI 0.62-0.88) (Table 2). Overall cancer mortality did not differ from the general population. However, mortality due to vulvar cancer was increased (SMR 28.1, 95\% CI 19.3-39.4) (Table 2).

\section{Discussion}

\section{Main Findings}

In this nationwide study, the age-adjusted incidence rate of LS rose to 22 per 100,000 woman-years in 2010-2012. The age-specific incidence of LS was highest in postmenopausal women, and was elevated in girls from five to nine years of age. The overall mortality of women with LS was lower than in the general Finnish female population; especially the risk of death from circulatory diseases, and dementia and Alzheimer's disease was decreased. 
The reduced mortality from these common causes outweighed the increase in mortality from vulvar cancer.

\section{Strengths and Limitations}

Studies on the epidemiology of LS are scarce. A strength of this study is the use of nationwide data gathered over a long period of time. These data were gathered from several registries, the quality of which has repeatedly been evaluated as good. ${ }^{12-14}$ Therefore, our study provides a significant improvement in generalizability over prior research.

The use of registry data also entails some limitations. Some patients with LS may have been left out of our cohort, because the Hospital Discharge Registry only includes diagnoses made in specialized health care including inpatient care from 1969, and outpatient care in public hospitals since 1998. Thus, patients diagnosed and treated in the primary health care or the private sector are not included, which biases the incidence estimates downwards and restricts the generalizability of our SMR estimates to all LS patients. The missing patients and the patients in the cohort may differ in several ways - e.g. in their health seeking behavior. On the other hand, the missing women may suffer from a milder disease not requiring assessment in specialized health care.

The LS diagnoses in the HDR may be clinical or histological. Therefore, our data may also include clinically misdiagnosed patients. However, according to the European guideline of LS, the diagnosis of LS is essentially clinical, and a biopsy is recommended in cases of uncertain diagnosis, treatment resistance, or suspected malignancy. ${ }^{10}$ Therefore, we estimate only few misdiagnosed patients within our cohort.

Some factors known to affect mortality are not collected into the registries used, thus their effects on the SMR could not be accounted for in this study. Such factors include smoking, alcohol consumption, body mass index, physical inactivity, and diet.

\section{Interpretation}


The incidence rate of LS found in the present study confirms that LS is not an uncommon disease. The dual-peaked incidence in prepubertal girls and postmenopausal women shown in previous small studies was now confirmed using population-based data. ${ }^{2,3}$

The frequency of LS in the population has been proven difficult to assess because patients underreport and physicians underrecognize it. Patients do not always seek medical care because they may be asymptomatic or have symptoms they do not wish to share with medical personnel. When they do seek medical attention, they present to different medical specialties but sometimes go undiagnosed still because the treating physician is unfamiliar with the disease.

The only previous study assessing the incidence of LS reported an age-adjusted (European Standard) rate of 16 per 100,000 woman-years in two provinces in the Netherlands in 20062011. ${ }^{6}$ However, that estimate only included histological diagnoses underestimating the true incidence of the disease. Our data are not restricted to histology-proven cases, which may explain the slightly greater incidence rate of 19 per 100,000 woman-years. Nevertheless, the true incidence rate may be higher still, since our data lack the mildest cases of LS as well as the asymptomatic, undiagnosed patients.

The Dutch study found a low incidence rate - i.e., 1.1 per 100,000 person-years - in girls in the age group from five to nine. ${ }^{6}$ Again, this is due to inclusion of only biopsy-proven cases of LS. The European guideline of LS discourages taking biopsies from girls with the exception of treatment-resistant or uncertain cases. ${ }^{10}$ Therefore, our incidence rate of 7 per 100,000 person-years in this age group is probably more accurate.

In the study by Bleeker et al., the incidence rate of LS nearly doubled from 9 per 100,000 woman-years in 1991-1995 to 16 per 100,000 woman-years in 2006-2011. ${ }^{6}$ We also found an increasing incidence rate over time, from 14 to 22 per 100,000 woman-years from 2003 to 2010-2012. One explanation for the increase may be that patients now are less reluctant to seek medical help for symptoms in the vulvar area, or physicians better diagnosing vulvar 
diseases. Another explanation could be changes in the environment over time, which may increase the incidence of the disease.

LS is known to have negative effects on patients' quality of life and sexual well-being. ${ }^{7,8}$ It also increases their risk of vulvar squamous cell carcinoma. ${ }^{9}$ In current literature, however, the effect of LS on mortality of patients has not been assessed before this study.

Inflammatory and some autoimmune diseases, such as rheumatoid arthritis (RA) and systemic lupus erythematosus (SLE), associate with an increased risk of death. ${ }^{15,16}$ LS causes local inflammation of the skin, which may be due to an autoimmune processes. Therefore, the decreased overall mortality of our LS women is somewhat unexpected. A healthier lifestyle is known to be associated with lower mortality and could explain this observation in our cohort. ${ }^{17-19}$ Deduced from the lower mortality due to lung cancer, bronchitis and emphysema, and alcohol-related causes we can assume that women in our LS cohort probably smoke less and consume less alcohol than the female population in general.

In an Italian study, patients with LS had higher educational attainment than that of the general population. ${ }^{20}$ Another Italian study found an association between parity and an increased risk of LS. ${ }^{21}$ If higher parity and educational status characterize also our patients, this would bias the mortality estimates downwards.

Another possible explanation for the overall reduction of mortality in patients with LS could be that they are followed within the health care system because of a chronic disease. Regular checkups may be a path to diagnosing and caring also for other diseases.

Cancer mortality of women living with LS did not differ from that of the general population, although autoimmune diseases, e.g. RA and SLE, are proven to affect patients' risk of cancer. ${ }^{22}$ In our LS cohort, an expected increase in mortality due to vulvar cancer was observed. On the contrary, the mortality due to cancers of lung, breast, and cervix were somewhat reduced. The risk of these cancers is related in part to lifestyle issues, ${ }^{23}$ and the aforementioned healthier lifestyle of our LS patients could explain this result. 


\section{Conclusion}

This study shows that the risk of getting LS by age 80 is $1.6 \%$. Physicians should maintain a high index of suspicion in postmenopausal women and girls with typical symptoms and signs. Fortunately, LS does not increase patients' mortality.

\section{Acknowledgements}

None.

\section{Disclosure of interests}

PH reports grants from the Finnish-Norwegian Medical Foundation and the Helsinki University Central Hospital Research funds. OH reports grants from the Helsinki University Central Hospital Research funds, Jenny and Antti Wihuri Foundation, Yrjö Jahnsson Foundation, and Finska Läkaresällskapet, during the conduct of the study; personal fees from Bayer Health Care AG, Gedeon-Richter, Sandoz AG, HRA-Pharma, and Vifor Pharma, outside the submitted work. The other authors have nothing to declare. Completed disclosure of interest forms are available to view online as supporting information.

\section{Contribution to authorship}

$\mathrm{PH}, \mathrm{MJ}, \mathrm{OH}, \mathrm{MG}$, and EP planned the design of the study. PH and EP participated in data analysis. PH wrote the first draft of the manuscript. All authors revised the manuscript and approved the final version.

\section{Details of ethics approval}

No ethics approval was needed, since this study is register-based, and no registered persons were contacted.

\section{Funding}

The Finnish-Norwegian Medical Foundation and the research funds of the Hospital system of Helsinki and Uusimaa supported this work. 


\section{References}

(1) Cooper SM, Ali I, Baldo M, Wojnarowska F. The association of lichen sclerosus and erosive lichen planus of the vulva with autoimmune disease: a case-control study. Arch Dermatol 2008 Nov;144(11):1432-1435.

(2) Wallace HJ. Lichen sclerosus et atrophicus. Trans St Johns Hosp Dermatol Soc 1971;57(1):9-30.

(3) Cooper SM, Gao XH, Powell JJ, Wojnarowska F. Does treatment of vulvar lichen sclerosus influence its prognosis? Arch Dermatol 2004 Jun;140(6):702-706.

(4) Goldstein AT, Marinoff SC, Christopher K, Srodon M. Prevalence of vulvar lichen sclerosus in a general gynecology practice. J Reprod Med 2005 Jul;50(7):477-480.

(5) Eberz B, Berghold A, Regauer S. High prevalence of concomitant anogenital lichen sclerosus and extragenital psoriasis in adult women. Obstet Gynecol 2008 May;111(5):11431147.

(6) Bleeker MC, Visser PJ, Overbeek LI, van Beurden M, Berkhof J. Lichen Sclerosus: Incidence and Risk of Vulvar Squamous Cell Carcinoma. Cancer Epidemiol Biomarkers Prev 2016 Aug;25(8):1224-1230.

(7) Van de Nieuwenhof HP, Meeuwis KA, Nieboer TE, Vergeer MC, Massuger LF, De Hullu JA. The effect of vulvar lichen sclerosus on quality of life and sexual functioning. J Psychosom Obstet Gynaecol 2010 Dec;31(4):279-284.

(8) Lansdorp CA, van den Hondel KE, Korfage IJ, van Gestel MJ, van der Meijden WI. Quality of life in Dutch women with lichen sclerosus. Br J Dermatol 2013 Apr;168(4):787-793.

(9) Halonen P, Jakobsson M, Heikinheimo O, Riska A, Gissler M, Pukkala E. Lichen sclerosus and risk of cancer. Int J Cancer 2017 May 1;140(9):1998-2002.

(10) Kirtschig G, Becker K, Gunthert A, Jasaitiene D, Cooper S, Chi CC, et al. Evidence-based (S3) Guideline on (anogenital) Lichen sclerosus. J Eur Acad Dermatol Venereol 2015 Oct;29(10):e1-43.

(11) Lee A, Bradford J, Fischer G. Long-term Management of Adult Vulvar Lichen Sclerosus: A Prospective Cohort Study of 507 Women. JAMA Dermatol 2015 Oct;151(10):1061-1067. (12) Pukkala E, Engholm G, Hojsgaard Schmidt LK, Storm H, Khan S, Lambe M, et al. Nordic Cancer Registries - an overview of their procedures and data comparability. Acta Oncol 2018 Apr;57(4):440-455. 
(13) Lahti RA, Penttila A. The validity of death certificates: routine validation of death certification and its effects on mortality statistics. Forensic Sci Int 2001 Jan 1;115(1-2):1532.

(14) Sund R. Quality of the Finnish Hospital Discharge Register: a systematic review. Scand J Public Health 2012 Aug;40(6):505-515.

(15) Dregan A, Chowienczyk P, Molokhia M. Cardiovascular and type 2 diabetes morbidity and all-cause mortality among diverse chronic inflammatory disorders. Heart 2017 Dec;103(23):1867-1873.

(16) Baena-Diez JM, Garcia-Gil M, Comas-Cufi M, Ramos R, Prieto-Alhambra D, SalvadorGonzalez B, et al. Association between chronic immune-mediated inflammatory diseases and cardiovascular risk. Heart 2018 Jan;104(2):119-126.

(17) Carter BD, Freedman ND, Jacobs EJ. Smoking and mortality--beyond established causes. N Engl J Med 2015 May 28;372(22):2170.

(18) Di Castelnuovo A, Costanzo S, Bagnardi V, Donati MB, Iacoviello L, de Gaetano G. Alcohol dosing and total mortality in men and women: an updated meta-analysis of 34 prospective studies. Arch Intern Med 2006 Dec 11-25;166(22):2437-2445.

(19) Loef M, Walach $H$. The combined effects of healthy lifestyle behaviors on all cause mortality: a systematic review and meta-analysis. Prev Med 2012 Sep;55(3):163-170. (20) Virgili A, Borghi A, Cazzaniga S, Di Landro A, Naldi L, Minghetti S, et al. New insights into potential risk factors and associations in genital lichen sclerosus: Data from a multicentre Italian study on 729 consecutive cases. J Eur Acad Dermatol Venereol 2017 Apr;31(4):699704.

(21) Sideri M, Parazzini F, Rognoni MT, La Vecchia C, Negri E, Garsia S, et al. Risk factors for vulvar lichen sclerosus. Am J Obstet Gynecol 1989 Jul;161(1):38-42.

(22) Giat E, Ehrenfeld M, Shoenfeld Y. Cancer and autoimmune diseases. Autoimmun Rev 2017 Oct;16(10):1049-1057.

(23) McKenzie F, Biessy C, Ferrari P, Freisling H, Rinaldi S, Chajes V, et al. Healthy Lifestyle and Risk of Cancer in the European Prospective Investigation Into Cancer and Nutrition Cohort Study. Medicine (Baltimore) 2016 Apr;95(16):e2850. 
Table 1. Number of lichen sclerosus patients by age at the beginning of follow-up, and person-years of follow-up by dynamic age.

\begin{tabular}{|l|c|c|}
\hline Age & Persons (\%) & Person-years (\%) \\
\hline $0-9$ & $206(2.6)$ & $710(1.0)$ \\
\hline $10-19$ & $120(1.5)$ & $1,780(2.6)$ \\
\hline $20-29$ & $204(2.6)$ & $1,519(2.2)$ \\
\hline $30-39$ & $383(4.9)$ & $5,522(3.7)$ \\
\hline $40-49$ & $798(10.2)$ & $11,075(16.1)$ \\
\hline $50-59$ & $1,631(20.9)$ & $17,475(25.4)$ \\
\hline $60-69$ & $2,030(26.1)$ & $17,202(25.1)$ \\
\hline $70-79$ & $1,733(22.2)$ & $10,878(15.8)$ \\
\hline $80+$ & $685(8.8)$ & $68,666(100)$ \\
\hline Total & $7,790(100)$ & \\
\hline
\end{tabular}


Table 2. Observed (OBS) and expected (EXP) numbers of deaths and standardized mortality ratios (SMR) with $\mathbf{9 5 \%}$ confidence intervals (CI) for selected causes of death of women with lichen sclerosus within a follow-up of more than five years.

\begin{tabular}{|c|c|c|c|c|}
\hline CAUSE OF DEATH & OBS & EXP & SMR & $95 \% \mathrm{CI}$ \\
\hline All & 766 & 910 & 0.84 & $0.78-0.90$ \\
\hline All diseases & 743 & 878 & 0.85 & $0.79-0.90$ \\
\hline Infections & 7 & 7 & 0.98 & $0.39-2.01$ \\
\hline Cancer & 183 & 178 & 1.03 & $0.89-1.18$ \\
\hline Lung, trachea & 12 & 20 & 0.60 & $0.31-1.04$ \\
\hline Breast & 18 & 24 & 0.74 & $0.44-1.17$ \\
\hline Genitals & 49 & 21 & 2.37 & $1.76-3.13$ \\
\hline Cervix uteri & - & 2 & 0.00 & $0.00-2.34$ \\
\hline Corpus uteri & 7 & 6 & 1.19 & $0.48-2.44$ \\
\hline Ovary & 7 & 10 & 0.72 & $0.29-1.47$ \\
\hline Vulva & 33 & 1 & 28.1 & $19.3-39.4$ \\
\hline Vagina & 2 & 0.4 & 5.50 & $0.67-19.9$ \\
\hline Skin, non-melanoma & 1 & 0.7 & 1.38 & $0.03-7.66$ \\
\hline $\begin{array}{l}\text { Endocrine, nutritional and metabolic } \\
\text { diseases }\end{array}$ & 7 & 10 & 0.68 & $0.27-1.39$ \\
\hline Diabetes mellitus & 7 & 8 & 0.83 & $0.33-1.71$ \\
\hline Dementia, Alzheimer's disease & 116 & 156 & 0.75 & $0.62-0.88$ \\
\hline Diseases of circulatory system & 329 & 410 & 0.80 & $0.72-0.89$ \\
\hline Ischemic heart diseases & 185 & 217 & 0.85 & $0.73-0.98$ \\
\hline Other heart diseases & 25 & 39 & 0.65 & $0.42-0.95$ \\
\hline Cerebrovascular diseases & 88 & 104 & 0.85 & $0.68-1.04$ \\
\hline Diseases of respiratory system & 35 & 36 & 0.98 & $0.68-1.35$ \\
\hline Bronchitis, emphysema & 7 & 12 & 0.59 & $0.24-1.22$ \\
\hline Diseases of digestive system & 21 & 27 & 0.77 & $0.48-1.17$ \\
\hline Diseases of genitourinary system & 9 & 10 & 0.89 & $0.41-1.69$ \\
\hline
\end{tabular}




\begin{tabular}{|l|r|r|r|c|}
\hline $\begin{array}{c}\text { Alcohol related diseases and accidental } \\
\text { poisoning by alcohol }\end{array}$ & 3 & 7 & 0.42 & $0.09-1.23$ \\
\hline Accidents and violence & 23 & 31 & 0.74 & $0.47-1.10$ \\
\hline Suicide & 2 & 3 & 0.63 & $0.08-2.26$ \\
\hline
\end{tabular}

This article is protected by copyright. All rights reserved 


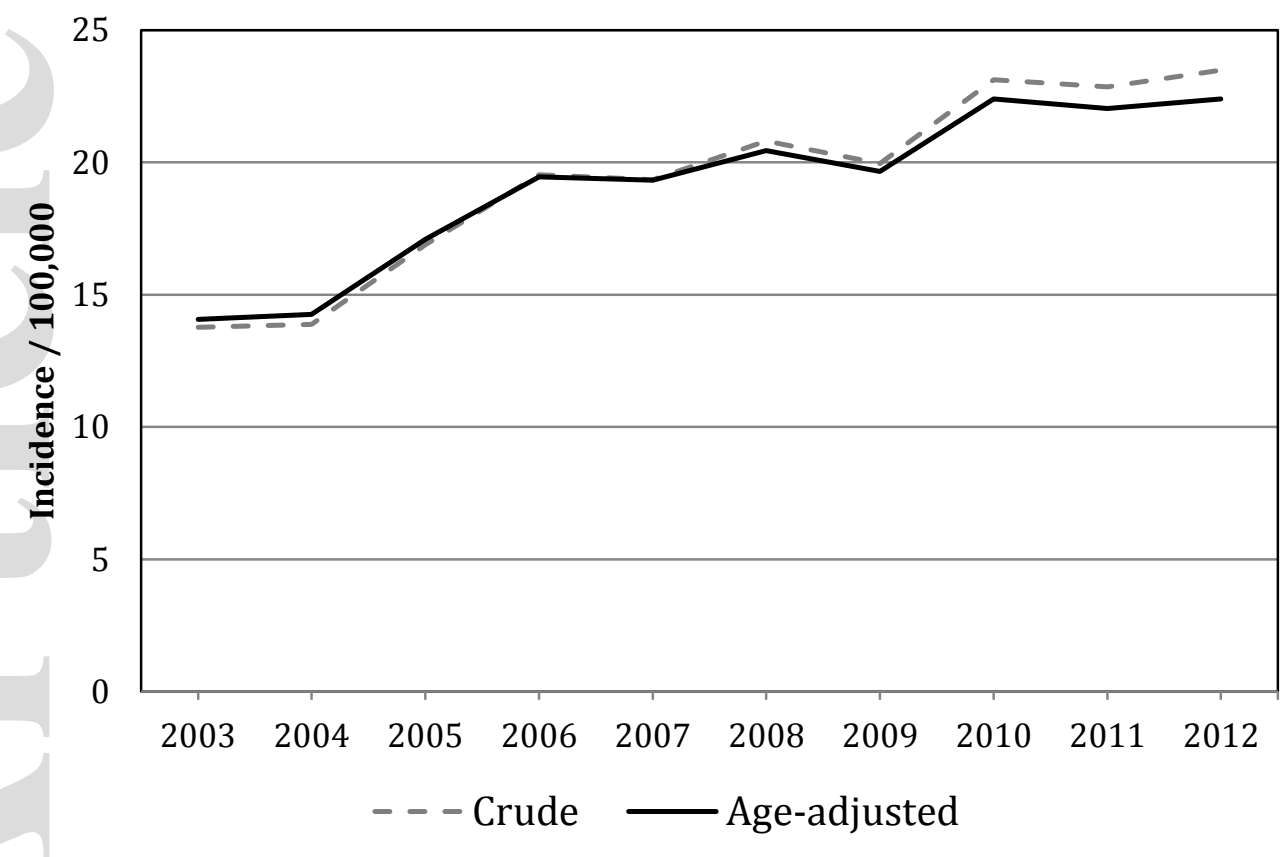

Figure 1. Crude and age-adjusted (European Standard population 2013) incidence rate of lichen sclerosus in Finland 2003-2012, per 100,000 person-years. 


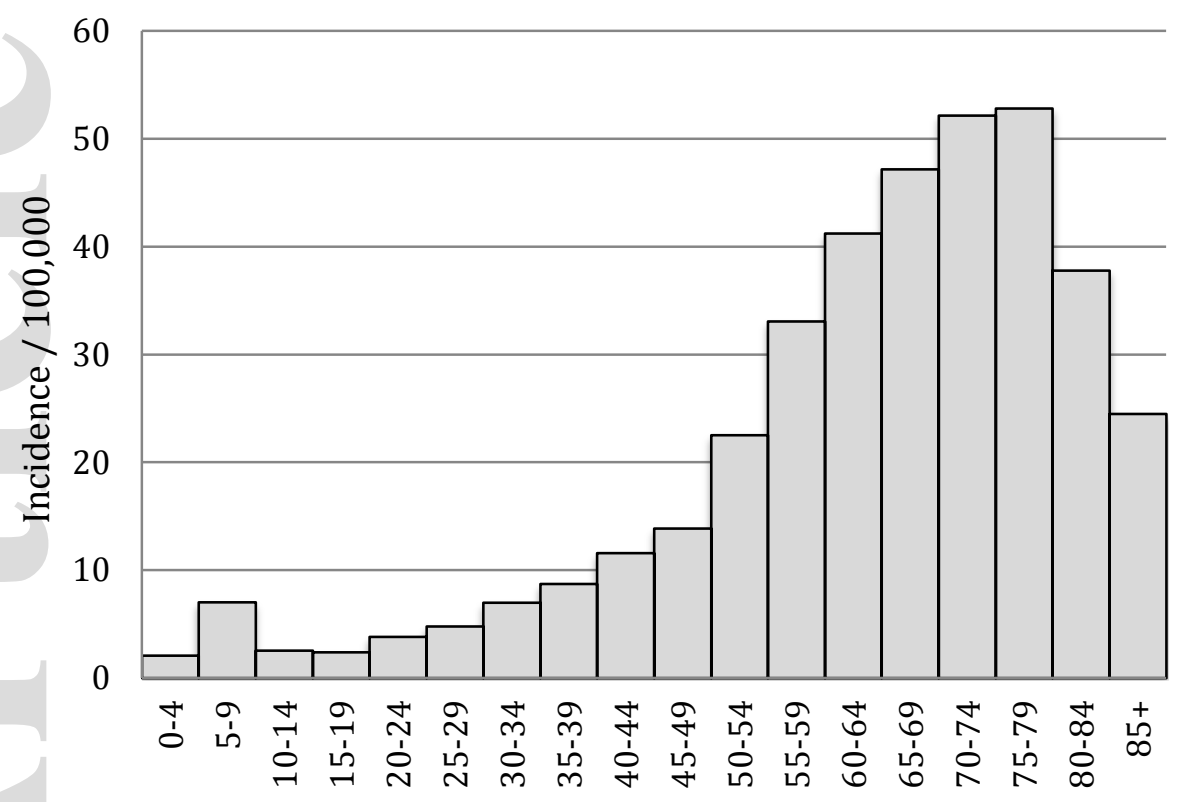

Figure 2. Age-specific incidence rates of lichen sclerosus in Finland 2003-2012, per 100,000 person-years. 